\title{
BMJ open Network structure and the role of key players in a translational cancer research network: a study protocol
}

\author{
Janet C Long, Frances C Cunningham, Jeffrey Braithwaite
}

To cite: Long JC, Cunningham FC, Braithwaite J. Network structure and the role of key players in a translational cancer research network: a study protocol. BMJ Open 2012;2: e001434. doi:10.1136/ bmjopen-2012-001434

- Prepublication history for this paper is available online. To view these files please visit the journal online (http:// dx.doi.org/10.1136/ bmjopen-2012-001430).

Received 7 May 2012 Accepted 31 May 2012

This final article is available for use under the terms of the Creative Commons Attribution Non-Commercial 2.0 Licence; see http://bmjopen.bmj.com

Centre for Clinical Governance Research, University of New South Wales, Kensington, New South Wales, Australia

Correspondence to Janet C Long; janet.long@ student.unsw.edu.au

\begin{abstract}
Introduction: Translational research networks are a deliberate strategy to bridge the gulf between biomedical research and clinical practice through interdisciplinary collaboration, supportive funding and infrastructure. The social network approach examines how the structure of the network and players who hold important positions within it constrain or enable function. This information can be used to guide network management and optimise its operations. The aim of this study was to describe the structure of a translational cancer research network (TCRN) in Australia over its first year, identify the key players within the network and explore these players' opportunities and constraints in maximising important network collaborations.
\end{abstract}

Methods and analysis: This study deploys a mixedmethod longitudinal design using social network analysis augmented by interviews and review of TCRN documents. The study will use network documents and interviews with governing body members to explore the broader context into which the network is embedded as well as the perceptions and expectations of members. Of particular interest are the attitudes and perceptions of clinicians compared with those of researchers. A co-authorship network will be constructed of TCRN members using journal and citation databases to assess the success of past pre-network collaborations. Two whole network social network surveys will be administered 12 months apart and parameters such as density, clustering, centrality and betweenness centrality computed and compared using UCINET and Netdraw. Key players will be identified and interviewed to understand the specific activities, barriers and enablers they face in that role. Ethics and dissemination: Ethics approvals were obtained from the University of New South Wales, South Eastern Sydney Northern Sector Local Health Network and Calvary Health Care Sydney. Results will be discussed with members of the TCRN, submitted to relevant journals and presented as oral presentations to clinicians, researchers and policymakers.

\section{INTRODUCTION}

\section{Translational research networks}

Notable even among other dynamic fields contributing to the scientific knowledge

\section{ARTICLE SUMMARY}

Article focus

n Translational research networks are a deliberate strategy to transform biomedical research 'at the bench' into clinical practice 'at the bedside' through interdisciplinary collaboration, supportive infrastructure, staff and funding

- What features of network structure hinder or promote successful translational research endeavours?

- What is the role of key players in a collaborative interdisciplinary network?

Key messages

- A protocol for a mixed method, longitudinal study of a new translational research network using a social network approach.

- A social network approach can inform management of the network and suggest ways in which to optimise its function.

Strengths and limitations of this study

- Mixed methods give quantitative data enriched with qualitative data.

- Data was collected from the whole network rather than from a sample of members.

- Longitudinal design gives comparative data rather than a snapshot.

explosion, biomedical research has greatly increased in output and complexity with the sequencing of the human genome and advances in sophisticated new technologies. ${ }^{1}$ As a result, increasingly specialised language, concepts and understandings have deepened the gulf between scientists and clinicians. Translational research is a deliberate strategy that seeks to transform biomedical research 'at the bench' into clinical practice 'at the bedside' through interdisciplinary collaboration and a range of supportive infrastructure, funding and staff. ${ }^{3-5}$

There are two distinct phases of translational research referred to as $\mathrm{T} 1$ and $\mathrm{T} 2$ by the Institute of Medicine's Clinical Round Table, each with unique goals, settings and participants. ${ }^{6}$ T1 refers to the interface 
between the laboratory and the clinical setting, striving to find how new knowledge of disease mechanisms can be developed into clinically relevant understandings, and diagnostic and treatment regimes to be trialled in humans. T2 seeks the translation of new clinically proven knowledge of disease processes, diagnostic or treatment techniques into routine clinical practice and health decision making. ${ }^{6}$ Others have suggested T3: practicebased research, ${ }^{8}$ where the evidence from clinical trials on carefully selected patients is translated into guidelines for complex patients seen routinely in practice.

Barriers to successful translation include structural, financial, cognitive and cultural factors. Structural barriers include the pressures of clinical service delivery, which have left many hospital or practice-based clinicians with insufficient time for research. ${ }^{6}{ }^{9-12}$ The unreliability of funding for translational research compounds this problem, ${ }^{10}{ }^{13}$ as does the lack of a recognised career path for translational researchers or clinician-scientists. ${ }^{2} 13$

Cognitive barriers include the need to understand unique research design in order to translate research generated in the highly controlled laboratory environment into the relatively chaotic and complex clinical environment. ${ }^{8} 12$ This may incorporate the use of experts such as biostatisticians to interpret results and information technology specialists to design and run more complex computer algorithms for integrated analyses. ${ }^{14}$ Furthermore, research carried out in the clinical setting requires the negotiation of a minefield of legal and regulatory requirements. ${ }^{13} 15$ Lack of standardisation of clinical trial protocols, data recording and evidence thresholds are also cited as barriers. ${ }^{6} 1416$ Inability to access results from previous trials or research programmes, especially those that have not reached publication, also hinders translation. ${ }^{101416}$

Most significant is the difference between the "two cultures ${ }^{10}$ : the contrasting paradigms, culture, expertise and language of basic science and clinical practice that need to be understood to prevent miscommunications, mismatched expectations and team conflict. ${ }^{9} 101317$ The divide is so significant that translational researchers may variously be seen as interpreters,${ }^{13}$ liaisons ${ }^{9}$ or conflict managers, ${ }^{18}$ in order to facilitate the collaboration.

Professional networks that bring together biomedical researchers and clinicians are believed to be an ideal vehicle to address the challenges of translational research allowing sharing of expertise, infrastructure and knowledge. In the USA, the National Centre for Advancing Translational Sciences has been established, complemented by the provision of dedicated grants for translational research (Clinical and Translational Science Awards) as well as a raft of recommendations and infrastructure support from the National Institutes of Health. ${ }^{451215}$ The UK has invested $£ 450$ million over 5 years to establish translational research centres including an Informatics Initiative. ${ }^{7} 12$ Australia also has invested in translational research initiatives such as
Academic Health Sciences Centres, ${ }^{19}$ and the funding of four new Translational Cancer Research Centres by the Cancer Institute of New South Wales. ${ }^{20}$

\section{The social network approach}

It is not clear how far funding incentives and streamlined infrastructure will take us in addressing the challenges inherent in translational research. An alternative approach is to look at translational collaborative initiatives through a social network lens. Social networks are defined as sets of people (actors) with relationships, ties or interactions between them. ${ }^{21}$ Translational research groupings are social networks with ties of communication and collaborative interactions between members. The focus of social network studies is the network's structure and how this relates to its function, for example, its efficiency of communication flows and resilience to change. Network structure provides the context for individuals' actions including the constraining and enabling factors that affect their behaviour. ${ }^{22}$ Certain positions within the network are highly influential and individuals who hold these positions are referred to key players.

A social network approach can inform management of the network and suggest ways in which to optimise its function. ${ }^{23}$ Moreover, attention to the role of key players in such networks may identify opportunities and barriers to improved network function and enable targeted interventions. ${ }^{18}{ }^{24}$ The aim of this study was to describe the structure of a translational cancer research network (TCRN) over the first year of its operation, identify the key players within the network and explore these players' opportunities and constraints in maximising important collaborative network functions.

The setting is a newly formed TCRN in New South Wales, Australia. The TCRN's stated aim is to develop a 'sustainable translational research engine' by providing leadership, co-ordination and infrastructure to a collaboration of 67 members made up of a variety of clinicians based primarily in hospitals, and university-based biomedical and health services researchers. The network has members from six different campuses and representation from a range of domains such as basic science, pathology, primary health, oncology and radiology. The TCRN officially commenced in July 2011. A project manager was appointed in September 2011, and actual project negotiations between members began in January 2012. Data collection for this study commenced in early 2012 at a time when there had been little official TCRN contact between most members. However, arising from a competitive grant process, the TCRN necessarily brought together a core of members who were known to one another and who had previously collaborated on research projects.

\section{Collaborative networks}

Translational research networks seek to set up conditions under which research can be optimally initiated and 
spread. Grouping members from different disciplines, sites and specialties, aiming to maximise knowledge transfer, ensuring co-ordination of activities and easy access to resources is a common theme in the translational research literature. ${ }^{3} 471012141625-27$ Collaborative networks are, of course, found across many other fields and in many contexts such as commercial, political and community, and there is a large body of literature considering various aspects of network structure and their mediating effects.

The customary tool for empirical measurement of network structure is social network analysis (SNA). While attribute data (eg, gender, age or years of experience) are usually collected in SNA, it is relational data that define the actual network structure. ${ }^{28}$ Ties need to be tightly defined as different relationships produce different networks. For example, the network defined by friendship may well be different from the network defined by reporting structure. This study will consider a number of different networks and characteristics: actual relationships such as past collaborations, people participants have worked with or know socially, as well as 'cognitive ties' or perceptions, ${ }^{29}$ such as intended future collaborations, or people they consider powerful or well-connected.

Some network parameters measured by SNA, used to describe the network's functioning, have been shown to affect network outcomes. These include the overall density and cohesion of network members, members' homophily and tendency to form discrete clusters within networks, the strength of ties between them, the gaps or structural holes between these clusters and the presence or absence of key players. The next section contains a discussion of these factors in terms of the research hypotheses they generate.

\section{Density and cohesion}

The density of the network is the number of links between members expressed as a proportion of the maximum possible number of links. ${ }^{28}$ Network density is a measure therefore of how connected members are. Densely linked networks are more efficient at diffusing information to all their members when compared with sparsely linked groups. ${ }^{30}$ Dense networks are also resilient to the loss of individual actors as there are enough alternate linkages to cover the loss. High density also prevents bottlenecks in advice-seeking or information networks. Cummings and Cross, ${ }^{31}$ in their study of 182 workgroups in a global business organisation, found that when actors had to go through their manager to get information or advice from an appropriate third party, productivity and efficiency suffered.

The TCRN is embedded in a pre-existing and complex interorganisational network of long-standing research and teaching arrangements as well as self-initiated collaborative research. TCRN activities and funded projects do not displace existing and ongoing research such as National Health and Medical Research
Council-funded projects. At its inception, the TCRN was already a collaborative effort with core group members who prepared and submitted the proposal to the funding body. Past collaborative ties and knowledge of a broad range of clinicians and researchers therefore define the starting point of the network and give a base line for growth in linkages of members and new collaborations over time.

Hypothesis 1: The TCRN's precursor collaboration network has a high density index.

Other network relationships considered are members they have worked with (but not collaborated with in translational research) and members they know socially (from outside of the work setting), referred to here as the work network and social network, respectively. It is expected that these ties will not be as frequent, and therefore, these networks will not be as dense as the precursor collaboration network.

Hypothesis 2: The TCRN's precursor collaboration network has a higher density than either the work network or the social network.

\section{Homophily and clusters}

Discrete areas of high density in a network are termed as clusters, and the gaps between them can slow or impede the flow of information or other resources within a network. Identifying the basis of clustering can be an important step in improving network function. ${ }^{32}$ The two cultures of researchers and clinicians, discussed above,${ }^{10}$ are the most obvious factors distinguishing members in a translational research network. Members will be asked to identify themselves as primarily 'clinician,' 'researcher' or the hybrid 'clinician-researcher.'

Hypothesis 3: Members of the TCRN cluster primarily around the 'two cultures' of academic research and clinical practice.

Strong as this factor may be, other factors may also be relevant. Homophily is typically associated with clustering and is defined by Rogers as "the extent to which two or more individuals who interact are similar in certain attributes." ${ }^{30}$ Attributes found to contribute to homophily apart from occupation include age, gender and level of education (see review by Brass et $a l^{33}$ ). Network analyses of health teams have shown strong clustering around individual professions. Creswick and colleagues $^{34-36}$ employed SNA on a renal ward and an emergency department of a large metropolitan hospital in Australia. They were able to show that across social advice-seeking and medication advice-seeking networks, there was clustering along professional lines: nursing, medical and allied health staff. West and Barron ${ }^{37}$ surveyed senior nurses and physician leaders in an acute care hospital in the UK. They found strong evidence to support their conjecture that clinicians' occupational 
networks demonstrate professional homophily. In the network based on ties with people with whom they discussed important professional matters, the clustering was strongest among their sample of doctors compared with the nurses, and homophily was also found on the basis of age, gender and level of experience. Similarly, studies of interdisciplinary research collaborations in university settings show clustering within research groups and disciplines. ${ }^{38} 39$

Clustering can also be associated with organisational structures. Actors employed doing the same tasks or in a similar hierarchical role have more reasons and opportunities to interact with each other. Cott ${ }^{40}$ showed two contrasting clusters in her analysis of decisionmaking teams in residential aged care: senior nurses and medical staff (those who 'decide') and junior and night staff nurses (those who 'carry out'). Geographic proximity (eg, working in the same hospital) ${ }^{37}$ and temporal alignment (eg, working the night shift or previously working together in the same place) ${ }^{40}$ also allow enhanced opportunities to interact and can result in clusters for certain types of interaction.

Attributes expected to contribute to homophily and clustering in the TCRN are specialty (meaning here the particular subarea of oncology research such as molecular biology, palliative care or radiotherapy), geographic location, seniority, profession and member's key tasks (such as direct patient care, supervision of students or laboratory work). It is noted that some of these factors overlap and differentiation of exact factors will be difficult, for example, key tasks and profession or seniority. However, it is expected that after the researcherclinician divide, the next most influential attribute will be specialty, based on the observation that wards, clinical services and research laboratories tend to be grouped this way.

Hypothesis 4: Clustering within the TCRN is also associated with specialty, and to a lesser extent, geographic location, seniority, profession and key tasks.

\section{Strength of ties}

Relationship ties can be weighted to reflect their strength, usually by assessing the frequency or intensity of contact. ${ }^{41}$ Strong ties have been associated with transfer of complex and tacit knowledge, ${ }^{42}$ and work teams' efficiency and stability over time. ${ }^{43}$ Strong ties are also associated with trust, another determinant of network success. ${ }^{44-46}$ Weak, in contrast to strong, ties imply less frequent contact and less invested time in the relationship. ${ }^{47}$ Strong ties have been associated with negative outcomes such as forming exclusive cliques that can impede communication within the network. TCRN members will be asked to weight the various relationships in their past collaboration, work and social networks as one of four strengths, from very weak to very strong. A fifth category, "I don't know this person at all" is used for the members not nominated. Again, given the antecedents of the TCRN, it is expected that past collaboration ties will be strong and work and social networks weak.

Hypothesis 5: The TCRN's past collaboration network has a high proportion of strong ties.

Hypothesis 6: The TCRN's work and social networks have a high proportion of weak ties.

Strong ties between members can also isolate the whole network from the broader industry and restrict the innovation that comes from access to new sources of information. ${ }^{414}$ It is now recognised that a combination of strong and weak ties are needed to maximise productivity, ${ }^{23}$ with a number of studies showing varying combinations of the two across a range of contexts (eg, see Jippes $e t a t^{49}$ and Ahuja $^{50}$ ).

\section{Key players}

SNA has allowed the identification of a range of actors that Borgatti terms key players ${ }^{51}$ : actors who have important roles within the network and are defined by their position within the overall structure. The two most common key players, central actors and bridging actors, are referred to by a confusingly wide range of names, some synonymous, others emphasising different aspects of the role across diverse settings. Actors who occupy central highly connected positions in the network are termed variously as central actors (eg, see Creswick et $a l^{36}$, Hawe and Ghali ${ }^{52}$ and McAneney et $a l^{53}$ ), opinion leaders (eg, see Valente ${ }^{54}$, Valente and Pumpuang ${ }^{55}$, Gifford et $a{ }^{56}$ and Ankem ${ }^{57}$ ), hubs (eg, see Buchanan ${ }^{58}$ and Watts and Strogatz $^{59}$ ) or connectors. ${ }^{60}$ Earlier sociometric studies called these people communication 'stars,' meaning actors who are chosen as friends by the most people ${ }^{61}$ or who are the individuals most approached for advice in a work setting. ${ }^{62}$ Central actors are able to co-ordinate activities and direct resources most efficiently due to their knowledge of and access to the most members in the network. ${ }^{47} 63$ Prestige and power are also often associated with central actors due to their unique position of control and access. Central actors who are said to have an opinion leading role have been shown to help dissemination of innovations ${ }^{49} 5664$ and to lead clinical quality and safety initiatives. ${ }^{65}$

Central actors in the TCRN are likely to be members of the core foundational group that initiated the funding proposal and so are also likely to be senior members of their specialty or profession. The clearest pattern of centrality is likely to be seen in the past collaboration network. It is expected that other members will perceive these central actors as 'powerful' or 'influential' individuals.

Hypothesis 7: Actors with high centrality in the past collaboration network are also perceived as powerful or influential actors by other members.

While clusters can show how people are grouped generally, a more precise definition of the gaps between 
groups of well-linked members is a 'structural hole'. A structural hole exists between two actors who are said to be non-redundant: that is between two actors who themselves are not connected. ${ }^{47}$ Contacts are said to be redundant to the extent that they lead to the same people and so provide the same information or opportunities. Non-redundancy therefore offers opportunities and information that can be accessed uniquely by the actor spanning the structural hole. Access to novel information is part of the mechanism behind generating 'good ideas' or innovations according to Burt, ${ }^{66} 67$ an important function of TCRNs. The inclusion of many different domains of cancer expertise in the TCRN is a deliberate strategy to facilitate this bridging of the gaps to produce innovative research ideas. Players who actually do the bridging will be brokering that potential innovation and are of particular interest to this study.

Key players who span structural holes have been given a wide variety of names, the commonest being bridges, ${ }^{47} 68$ boundary spanners or riders, ${ }^{69}$ or brokers. ${ }^{70-72}$ Gould and Fernadez, ${ }^{71}$ looking at what they call transaction networks, divided the role of the boundary spanner into five categories each reflecting a unique role, motivation or context. For example, gatekeepers are actors who bridge the structural hole between their cluster and an outside cluster, controlling what information passes into or out of their cluster. These categories have been expanded by Shi and colleagues $^{72}$ to eight categories to include a hierarchical dimension. Cross and Prusak ${ }^{70}$ divide the roles differently again: central connectors, peripheral specialists, boundary spanners and information brokers.

A focus of this study is to understand the role and associated activities of key players within translational research networks. Gray, ${ }^{18}$ considering the role of a transdisciplinary researcher, conceptualises the bridging role as one of conflict resolution. The importance of understanding concepts and language from both sides of the structural hole is seen as the key to facilitating collaboration across the divide.

Bridges within the TCRN will be those members who form a link between existing clusters. Since it is hypothesised that the main basis for clusters or structural holes is the researcher-clinician divide, members who specialise in clinical research and who have a so-called 'foot in each camp' are the most likely bridges.

Hypothesis 8: Actors identified as being bridges across structural holes in the TCRN are more likely to define themselves as clinician-researchers.

Some TCRN members are the nominated representatives of an outside work group or service that may provide information, expertise or resources relevant to TCRN activities. Others are planning to be the link between the TCRN and their colleagues, workplace or association to disseminate TCRN findings most effectively. Since the TCRN is embedded in the complex interorganisational structure of the NSW health system, all members are expected to be members of some common outside groups. However, the gap between the TCRN and other non-TCRN groups can be considered a structural hole if the TCRN member spanning the gap is the only link. Such links to outside groups has been strongly linked to innovative output. Shan and colleagues, ${ }^{73}$ considering the gap between biotechnology companies as a structural hole, found that the number of bridging ties that the company had with other companies was positively related to its innovation output. Ahuja ${ }^{50}$ found the same relationship in the pharmaceutical industry, but only up to a point; beyond which increasing the number of structural holes led to a decrease in innovative output.

The broader reach of the TCRN will be assessed by asking members to name outside groups to which they plan to disseminate TCRN findings and activities, or whom they represent (such as a service or owner of a particular resource). Members who are involved with many outside groups are often perceived as 'wellconnected' and can be labelled 'cosmopolites' (from the adjective cosmopolitan) and may be approached as a go-between or liaison, another bridging role.

Hypothesis 9: Actors identified as being bridges from the TCRN to relevant outside groups are perceived as well-connected members.

\section{METHOD/DESIGN}

This study is a mixed-method longitudinal study of a TCRN designed in seven phases. It uses documentary evidence, interviews, two online social network surveys 12 months apart and citation databases to examine the broader context into which the TCRN is embedded, the unique features of the network and how it changes in structure over the initial 12 month period, as well as focusing on the specific activities and perceptions of its key players.

\section{Phase 1}

A systematic review of the empirical peer-reviewed literature on social-professional networks and key network players was undertaken to inform the design of the study (Long JC, Cunningham FC, Braithwaite J. Brokers in social-professional networks: a systematic review, 2012, in preparation).

\section{Phase 2}

In this phase, the context of the network will be described. The research manager will be interviewed and documents produced by the TCRN will be reviewed. This will provide an understanding of the network's responsibilities towards the funding body, their formal objectives and outcomes and the rationale behind administrative decisions such as the composition of support staff, choice of initial membership and investment in infrastructure. Demographic information about 
members such as job title, primary place of work and profession will be sourced from the network's documentation.

\section{Phase 3}

This will consist of semistructured interviews with the 13 members of the Governing Council. Questions seek to elicit information about the wider translational research context into which the TCRN is being introduced. Participants will be asked to give information and opinions on the human and social capital they bring to the network, their perceptions of their role within the network, of how they will measure success, and of the challenges to producing satisfactory outcomes. Participants will also be asked to speculate on the basis of any clusters that might be revealed through SNA. Differences in network expectations and perceptions between hospital-based and university-based participants will be of particular interest. Participants will also be asked about past collaboration with other TCRN members. These responses will help to inform design of the social network survey.

\section{Phase 4}

This phase will start with the piloting of the online social network survey on 10 members of equivalent clinical or research experience in another network. Feedback from the pilot will be used to refine and clarify the survey before all members of the TCRN are invited to complete it with each member receiving a link to the secure survey site via personal email. The survey is a whole network survey; ${ }^{745}$ that is, all members are being surveyed rather than a sample of members. Therefore to maximise the response rate, two follow-up reminders will be emailed to non-respondents at 2 and 4 weeks.

The survey is divided into two sections. The first section collects demographic information (not able to be sourced from TCRN documents), which is hypothesised to be associated with clustering: key tasks, seniority, past or present places of work or tertiary study and membership in other networks. As some members have a clinical role as well as a research role, it is anticipated that the concept of the 'two cultures' or clinician or researcher may be unclear. Members will therefore be asked whether they identify more with the title clinician, researcher or clinical-researcher and their answer compared with other demographic information they give.

The members' perceptions of the TCRN and their role within it will be explored with a series of questions. Check box answers will be used to collect information on members' method of joining the network (self-initiated, by invitation or required by a superior) and motives for joining (answers reflecting an altruistic and patientcentred attitude, an organisation-centred or professioncentred attitude or a more opportunistic approach). Respondents will be asked if their role in the network includes representing their work group, their place of work or their profession and will be given an opportunity to name the group represented. Likewise, respondents who indicate that part of their role is the dissemination of TCRN findings and activities will be asked to nominate the groups they are likely to target and the number of people they could potentially reach. As well as role perceptions these questions give an indication of the potential reach of the TCRN into a wider setting. Members will be asked about challenges facing the success of the network answering first in free text and then choosing from a list of barriers and facilitators drawn from the literature and phase 3 interviews.

The second section of the survey contains a roster of members' names, grouped by primary place of work and asks two sets of questions that seek to ascertain the number and strength of interactions between network members before the TCRN formed, and interactions that formed or were intending to be formed as a direct result of the TCRN. Respondents will be asked to nominate members with whom they had previously collaborated, worked or knew socially (three parallel questions). Choosing one of four relationship categories will indicate the strength of each of these nominated relationships: weak (no longer in contact), medium (infrequent or superficial contact), strong (frequent or purposeful contact) or long-standing (long-term, stable relationship irrespective of frequency of contact). The second set of social network questions will ask about intended future collaborations as part of the TCRN and perceptions of powerful, influential and well-connected members.

\section{Phase 5}

A co-authorship network will be constructed where links in the network are defined by whether members have authored a paper with another member. This will give an indication of the success of previous collaborations among the TCRN members. Members' names will be searched for individually within Scopus and Web of Science citation databases from 1980 till April 2012 and articles downloaded into an Endnote library (an electronic referencing programme). Grey literature will be included as well as peer reviewed journal articles (but will be able to be differentiated in the network). After removing duplicates, co-authors will be identified and each collaboration recorded as a tie between those members. Published output will be compared to the collaborations nominated in the social network survey as a measure of their success.

\section{Phase 6}

In this phase, up to five key players identified from phase 4 will be interviewed. Members' roles will be explored, in-depth: their perceptions of how the network functions and their role in it, including specific details on their activities and experience of constraining and facilitating factors for those activities.

\section{Phase 7}

Finally, in phase 7 the online social network survey will be re-administered to all members of the network 
12 months after the first survey. It is expected that by this time, the number of network members will have at least doubled.

\section{ANALYSES}

Interviews will be recorded, transcribed and analysed with the assistance of NVivo9, a software package. Themes will be coded by two independent reviewers and finalised using an iterative process of discussion.

Social networks (with weighted relations), current and future collaboration networks and the co-authorship networks will be analysed using UCINET V.6 software ${ }^{76}$ and NetDraw will be used to map the networks graphically. ${ }^{77}$ Whole network parameters such as density, mean path length and centrality will be computed. Changes between the network at time 1 and time 2 will be evaluated. Network graphics will be used to visually explore the networks. Demographic data will be used to determine the basis of clustering. Central actors will be identified by mean degree (number of ties as a proportion of the number of actors), global centrality (the most connected actors within the network) ${ }^{78}$ and betweenness centrality (the extent to which an actor lies between actors that would not otherwise be connected) ${ }^{78}$ Bridges that span structural holes will identified by betweenness centrality $^{78}$ and bridging. ${ }^{68}$ Individual actors and sites will all be de-identified for presentation.

\section{ETHICS AND DISSEMINATION}

This project is part of the ARC Discovery Project DP0986493: 'Evaluating communities of practice and social-professional networks: The development, design, testing, refinement, simulation and application of an evaluation framework'. Ethics approvals were sought and obtained from the following organisations: University of New South Wales (HREC reference no: 09085), appropriate local health network and site-specific committees.

Results will be presented and discussed with members of the TCRN with a view to providing insights into the network's function and optimisation. In addition, results will be submitted to relevant journals and presented as oral presentations to clinicians, researchers and policymakers.

Acknowledgements We thank Professor Robyn Ward for her invaluable comments on the manuscript. The Translational Cancer Research Centre Scheme is supported by the Cancer Institute of New South Wales.

Contributors Protocol developed by all three authors. JCL wrote the paper, and FCC and JB critically reviewed all drafts and final copy.

Funding This work was supported by ARC Discovery Project (grant number DP0986493).

Competing interests None.

Ethics approval Ethics approval was provided by the University of New South Wales, appropriate local health network and site-specific committees.

Provenance and peer review Not commissioned; externally peer reviewed.

\section{REFERENCES}

1. Nicogossian A, Kloiber O, Zimmerman T, et al. Translational research, evidence-based medicine and medical policy. World Med Health Policy 2010;2. http://www.psocommons.org/wmhp/vol2/iss4/ art1 (accessed 12 Apr 2012).

2. Schwartz K, Vilquin JT. Building the translational highway: toward new partnerships between academia and the private sector. Nat Med 2003:9:493-5.

3. Goldblatt EM, Lee WH. From bench to bedside: the growing use of translational research in cancer medicine. Am J Transl Res 2010;2:1-18.

4. Zerhouni EA. Translational and clinical science: time for a new vision N Engl J Med 2005;353:1621-3.

5. National Institutes of Health. Translational Research: Overview. 2011. http://commonfund.nih.gov/index.aspx (accessed 12 Sep 2011).

6. Sung NS, Crowley WF, Genel M, et al. Central challenges facing the national clinical research enterprise. JAMA 2003;289:1278-87.

7. Woolf $\mathrm{SH}$. The meaning of translational research and why it matters. JAMA 2008;299:211-13.

8. Westfall JM, Mold J, Fagnan L. Practice-based research-"blue highways" on the NIH roadmap. JAMA 2007;297:403-6.

9. Bristow R. Recommendations for the future of translational radiobiology research: a Canadian perspective. Radiother Oncol 2004;70:159-64.

10. Dauphinee D, Martin JB. Breaking down the walls: thoughts on the scholarship of integration. Acad Med 2000;75:881-6.

11. Mankoff S, Brander C, Ferrone S, et al. Lost in translation: obstacles to translational medicine. J Transl Med 2004;2:14.

12. Tageja N. Bridging the translation gap-new hopes, new challenges Fundam Clin Pharmacol 2011;25:163-71.

13. Carpenter S. Carving a career in translational research. Science 2007;317:966-7.

14. Mathew JP, Taylor BS, Bader GD, et al. From bytes to bedside: data integration and computational biology for translational cancer research. PLoS Comput Biol 2007;3:e12.

15. Marantz PR, Strelnick AH, Currie B, et al. Developing a multidisciplinary model of comparative effectiveness research within a clinical and translational science award. Acad Med 2011;86:712-17.

16. Field A, Baxter K, Terry SF. From bench to practice to population health impact: barriers to realizing the public health and clinical promise of basic scientific discovery. Genet Test Mol Biomark 2011;15:191-2

17. Ioannidis JP. Materializing research promises: opportunities, priorities and conflicts in translational medicine. J Transl Med 2004;2:5-10.

18. Gray B. Enhancing transdisciplinary research through collaborative leadership. Am J Prev Med 2008:35:S124-32.

19. Daly J, Davidson PM, Duffield C, et al. Interdisciplinary, crossinstitutional collaborations: the Academic Health Sciences Centre as a key to addressing complex health problems and advancing research based health care. Collegian 2011;19:1-2.

20. Cancer Institute NSW. Translational Cancer Research Centres in NSW. 2012. http://www.cancerinstitute.org.au/research-grants-andfunding/translational-cancer-research-centres (accessed $1 \mathrm{Apr}$ 2012).

21. Newman MEJ, Watts DJ, Strogatz SH. Random graph models of social networks. Proc Natl Acad Sci U S A 2002;19:2566-72.

22. Emirbayer M, Goodwin J. Network analysis, culture, and the problem of agency. Am J Sociol 1994;99:1411-54.

23. Chauvet V, Chollett B, Soda $\mathrm{G}$, et al. The contribution of network research to managerial culture and practice. Eur Manag $J$ 2011;29:321-34.

24. Haines V, Godley J, Hawe P. Understanding interdisciplinary collaborations as social networks. Am J Community Psychol 2011;47:1-11.

25. Tenenbaum JD, Whetzel PL, Anderson K, et al. The Biomedical Resource Ontology (BRO) to enable resource discovery in clinical and translational research. J Biomed Inform 2011;44:137-45.

26. Chabner BA, Boral AL, Multani P. Translational research: walking the bridge between idea and cure: 17th Bruce F. Cain memorial award lecture. Cancer Res 1998:58:4211-16.

27. Bartlett $\mathrm{RH}$. Translating innovation: Beethoven, Gross, Krummel, and Georgeson. J Pediatr Surg 2011;46:18-21.

28. Scott J. Social Network Analysis: A Handbook. 2 edn. London: Sage, 2000.

29. Balkundi $P$, Kilduff $M$. The ties that lead: a social network approach to leadership. Leadersh Q 2005;16:941-62.

30. Rogers E. Diffusion of Innovations. 4 edn. New York: Free Press, 2003.

31. Cummings JN, Cross R. Structural properties of work groups and their consequences for performance. Soc Netw 2003;25:197-210. 
32. Braithwaite J. Between group behaviour in health care: gaps, edges, boundaries, disconnections, weak ties, spaces and holes. A systematic review. BMC Health Serv Res 2010;10:330.

33. Brass DJ, Galaskiewicz J, Greve HR, et al. Taking stock of networks and organizations: a multilevel perspective. Acad Manage $J$ 2004:47:795-817.

34. Creswick N, Westbrook JI. The medication advice-seeking network of staff in an Australian hospital renal ward. Inf Technol Health Care 2007; 130:217-31.

35. Creswick N, Westbrook JI. Social network analysis of medication advice-seeking interactions among staff in an Australian hospital. Int $J$ Med Inform 2010;79:e116-25.

36. Creswick N, Westbrook JI, Braithwaite J. Understanding communication networks in the emergency department. BMC Health Serv Res 2009;9:247.

37. West E, Barron DN. Social and geographical boundaries around senior nurse and physician leaders: an application of social network analysis. Can J Nurs Res 2005;37:132-48.

38. Mosey S, Westhead P, Lockett A. University technology transfer: network bridge promotion by the medici fellowship scheme. J Small Bus Enterprise Dev 2007;14:360-84.

39. Weng C, Gallagher D, Bales M, et al. Understanding Interdisciplinary Health Sciences Collaborations: A Campus-Wide Survey Of Obesity Experts. AMIA 2008 Symposium Proceedings, 2008. http://www.ncbi.nlm.nih.gov/pmc/issues/177327/ (accessed 12 Apr 2012).

40. Cott C. "We decide, you carry it out": a social network analysis of multidisciplinary long-term care teams. Soc Sci Med 1997;45:1411-21.

41. Granovetter M. The strength of weak ties. Am J Sociol 1973;78:1360-80.

42. Hansen M. Knowledge networks: explaining effective knowledge sharing in multiunit companies. Organ Sci 2002;13:232-48.

43. Balkundi P, Harrison DA. Ties, leaders and time in teams: strong inference about network structure's effects on team viability and performance. Acad Manage J 2006;49:49-68.

44. Zaheer A, McEvily B, Perrone V. Does trust matter? Exploring role interorganizational interpersonal trust performance. Organ Sci 1998;9:141-59.

45. Coleman JS. Social capital in the creation of human capital. Am J Sociol 1988;94:S95-120.

46. Lambright $\mathrm{KT}$, Mischen PA, Laramee CB. Building trust in public and nonprofit networks: personal, dyadic, and third-party influences. Amer Rev Public Adm 2010;40:64-82.

47. Burt RS. Structural Holes: The Social Structure Of Competition. Cambridge, Massachusetts: Harvard University Press, 1992.

48. Uzzi B. Social structure and competition in interfirm networks: the paradox of embeddedness. Adm Sci Q 1997;42:35-67.

49. Jippes E, Achterkamp MC, Brand PLP, et al. Disseminating educational innovations in health care practice: training versus social networks. Soc Sci Med 2010;70:1509-17.

50. Ahuja G. Collaboration networks, structural holes, and innovation: a longitudinal study. Adm Sci Q 2000;45:425-57.

51. Borgatti SP. Identifying sets of key players in a social network. Computational Math Organ Theory 2006;12:21.

52. Hawe P, Ghali L. Use of social network analysis to map the social relationships of staff and teachers at school. Health Educ Res 2008:23:62-9.

53. McAneney H, McCann J, Prior L, et al. Translating evidence into practice: a shared priority in public health? Soc Sci Med 2010;70:1492-500.
54. Valente T. Opinion leader interventions in social networks. BMJ 2006;333:1082-3

55. Valente T, Pumpuang P. Identifying opinion leaders to promote behavior change. Health Educ Behav 2007;34:881-96.

56. Gifford D, Holloway R, Frankel M, et al. Improving adherence to dementia guidelines through education and opinion leaders. Ann Intern Med 1999;131:237-46.

57. Ankem K. Influence of information sources on the adoption of uterine fibroid embolization by interventional radiologists. J Med Libr Assoc 2003;91:450-9.

58. Buchanan M. Nexus: Small Worlds and The Groundbreaking Science Of Networks. New York: WW Norton, 2003.

59. Watts DJ, Strogatz SH. Collective dynamics of 'small-world' networks. Nature 1998;393:440-2.

60. Gladwell M. The Tipping Point: How Little Things Can Make a Big Difference. New York: Back Bay Books / Little, Brown and Company 2000.

61. Moreno JL, Jennings HH. Who shall survive? A new approach to the problem of human interrelations. Washington, D.C.: Nervous and Mental Disease Publishing Co., 1934.

62. Allen TJ. Communication networks in R \& D labs. $R D$ Manag 1970;1:14-21.

63. Provan KG, Milward HB. A preliminary theory of interorganizational network effectiveness: a comparative study of four community menta health systems. Adm Sci Q 1995;40:1-33.

64. Lee SH, Cotte J, Noseworthy TJ. The role of network centrality in the flow of consumer influence. J Consumer Psychol 2009;20:66-77.

65. Hayes $\mathrm{C}$, Yousefi V, Wallington $\mathrm{T}$, et al. Case study of physician leaders in quality patient safety, and the development of a physician leadership network. Healthc Q 2010;13:68-73.

66. Burt RS. Structural holes and good ideas. Am J Sociol 2004;110:349-99.

67. Burt RS. Brokerage And Closure: An Introduction To Social Capital. New York: Oxford University Press, 2005.

68. Valente T, Fujimoto K. Bridging: locating critical connectors in a network. Soc Netw 2010;23:212-20.

69. Tushman ML. Special boundary roles in the innovation process. Adm Sci Q 1977;22:587-605.

70. Cross R, Prusak L. The people who make organizations go-or stop. Harv Bus Rev 2002;80:105-12.

71. Gould RV, Fernandez RM. Structures of mediation: a formal approach to brokerage in transaction networks. Sociol Methodol 1989;19:89-126.

72. Shi W, Markoczy L, Dess GG. The role of middle management in the strategy process: group affiliation, structural holes, and tertius iungens. J Manag 2009;35:1453-80.

73. Shan W, Walker G, Kogut B. Interfirm co-operation and startup innovation in the biotechnology industry. Strategic Manag $J$ 1994;15:387-94.

74. Wasserman S, Faust K. Social Network Analysis. Cambridge: Cambridge University Press, 1994.

75. Friedkin NE. The development of structure in random networks: an analysis of the effects of increasing network density on five measures of structure. Soc Netw 1981;3:41-52.

76. Borgatti SP, Everett MG, Freeman LC. UCINET for Windows: Software For Social Network Analysis. 6th edn. Harvard: Analytic Technologies, 2002.

77. Borgatti SP. NetDraw: graph visualization software. Harv Analytic Technologies 2002.

78. Freeman LC. Centrality in social networks: a conceptual clarification Soc Netw 1979;1:215-39. 